\title{
Quick and Simple Spectrophotometric Method of Identification of the Thermal State of Meat on the Basis of Composition and Content of Free Nucleotides
}

\author{
Magomed Dibirasulaev1, Georgy Belozerov¹, Leonid Arkhipov1, Lidia Stoyanova² \\ ${ }^{1}$ All-Russian Scientific Research Institute of Refrigeration Industry, Branch of V.M. Gorbatov Federal Research Center for Food \\ Systems of Russian Academy of Science, Moscow, Russia \\ ${ }^{2}$ M. V. Lomonosov Moscow State University, Moscow, Russia \\ Email: stoyanovamsu@mail.ru
}

How to cite this paper: Dibirasulaev, M., Belozerov, G., Arkhipov, L. and Stoyanova, L. (2018) Quick and Simple Spectrophotometric Method of Identification of the Thermal State of Meat on the Basis of Composition and Content of Free Nucleotides. Food and Nutrition Sciences, 9, 572-583.

https://doi.org/10.4236/fns.2018.95043

Received: April 17, 2018

Accepted: May 20, 2018

Published: May 23, 2018

Copyright $\odot 2018$ by authors and Scientific Research Publishing Inc. This work is licensed under the Creative Commons Attribution International License (CC BY 4.0).

http://creativecommons.org/licenses/by/4.0/

\section{(c) (i) Open Access}

\begin{abstract}
At present, there is no method for identifying meat frozen in a thermal state acceptable for production control. The role of free nucleotides in the processes of refrigeration and storage of meat, affecting the structural and mechanical properties of muscle tissue, the formation of taste and its biological value is known. In this article we compared methods for identifying the thermal state of meat based on the determination of the composition and content of free nucleotides by high-performance liquid chromatography (HPLC) and spectrophotometry [SF]. High-purity reference substances were used: free nucleotides-ATP, ADP, AMP, IMP and nucleosides-inosine and hypoxanthine. It has been experimentally established that the characteristic peaks of the absorption spectra for extracts of free nucleotides of meat frozen depended from thermal state of meat. The content of ATP is 21.8 times higher in meat frozen in a fresh state, and the amount of IMF is 12.3 times lower than in meat frozen after cooling. The results of studies of meat frozen using the HPLC method and the developed SF method show the adequacy of the data obtained by both methods. SF-method based on the determination of the optical density of the extracts of free nucleotides is recommended to justify the choice of technological process meat defrost modes.
\end{abstract}

\section{Keywords}

Thermal State of Meat, Free Nucleotides and Nucleosides, HPLC and Spectrophotometric Methods, Defrost Modes 


\section{Introduction}

Freezing is one of the most common ways to preserve the quality of meat and extend its shelf life. The initial thermal state of the raw material before freezing has a significant effect on the quality of meat and the loss of its mass during thawing. According to the thermal state, the meat is divided into: fresh-obtained immediately after slaughter; frozen - fresh or chilled meat subjected to refrigerating treatment to a temperature in the thickness of the muscle at a depth of 1 $\mathrm{cm}$ from $-3^{\circ} \mathrm{C}$ to $-5^{\circ} \mathrm{C}$ at a depth of $6 \mathrm{~cm}$ from $0^{\circ} \mathrm{C}$ to $2^{\circ} \mathrm{C}$, With storage temperature over the entire volume must be between minus $2^{\circ} \mathrm{C}$ to minus $3^{\circ} \mathrm{C}$.

Frozen-steamed, cooled or chilled meat, frozen to a temperature in the thickness of the muscles not above minus $8^{\circ} \mathrm{C}$. Deep freezing-frozen meat with a temperature in the thickness of the muscles is not above minus $18^{\circ} \mathrm{C}$. Thawed-frozen meat, heated to a temperature in the thickness of the muscles not below minus $1^{\circ} \mathrm{C}$ cooled down-carcasses obtained immediately after slaughter and processing, having a temperature in the muscle thickness of no higher than $12^{\circ} \mathrm{C}$ [1] [2] [3]. In Russia, the production of frozen blocks from meat is carried out from chilled raw materials, in the world, for example, in New Zealand 40\% of all meat produced is frozen in a fresh state in the form of boneless meat cuts [4].

In the majority of works the special role of nucleotides in the biochemical processes occurring in meat after slaughter of an animal is noted. They are described as a precursor to the formation of compounds that give the meat a specific taste and aroma. It is believed that the quantitative content, nature and speed of changes in the qualitative composition of nucleotides in meat, significantly affect the formation of physico-chemical properties and quality characteristics of raw meat, its nutritional and biological value. Qualitative composition and quantitative content of free nucleotides and nucleosides of meat at various stages of refrigeration and storage is changed. After 24-h storage of fresh meat content ATP is almost exhausted and there is an accumulation of lactic acid. Enzymatic breakdown of glycogen provides the start-up of subsequent physico-chemical and biochemical processes [5] [6]. When freezing meat, physico-chemical, structural-mechanical, biochemical changes take place, the depth and degree of manifestation of which is determined by: the freezing speed, the depth of autolytic changes in meat raw material at the time of freezing, and the process of crystal formation of water in meat [7]. Muscles and connective tissues give the meat a certain structure. Immediately after the slaughter, the meat retains the fibrous structure for as long as the reserves of ATP (adenosine triphosphate) in the muscles allow. The quality of meat after the end of storage in a frozen form depends on the degree of autolysis before freezing. When freezing fresh meat (single-phase method), the activity of the enzymes is well preserved, with the thawing of such raw materials, maturation is similar to fresh meat. However, during the defrosting of meat subjected to a two-phase freezing, significant autolytic destruction of many microstructures is observed, which is due 
to high mass losses during defrosting and low flavor and nutritional indices [8] [9] [10] [11].

In order to preserve the quality and reduce the loss of weight during thawing of meat frozen in a fresh form or after cooling, differentiated defrosting technologies should be applied. However, to date, there is no quick method for identifying meat frozen in a batch form or after cooling, acceptable for production control. The studies carried out to date on the role of free nucleotides in the processes of ripening, refrigeration and storage of meat show their participation in mechanochemical processes, the formation of taste and changes in the structural and mechanical properties of muscle tissue [9].

As a result of previous studies on determining the composition and content of free nucleotides during refrigeration and storage of meat, a method for determining the number of stages (single-phase or two-phase) that the meat was subjected to during freezing was developed and patented [1].

However, this method requires the use of a high-pressure liquid chromatograph, which makes it difficult to use it for industrial control at enterprises.

The goal of this work is the development of a spectrophotometric method based on the determination of the optical density of extracts of free nucleotides of muscle tissue for the accelerated identification of the thermal state of meat frozen in a fresh state or after cooling.

\section{Materials and Methods}

The muscles of cattle of the first category (the longest muscle of the back of Longissimus dorsi) were used for the study, in the fresh state (30 - 45 minutes after slaughter with a temperature in the muscle mass of $35^{\circ} \mathrm{C}-39^{\circ} \mathrm{C}$ ) and the cooled state (24 hours after slaughter with temperature in the thickness of the muscles $2^{\circ} \mathrm{C} \pm 2{ }^{\circ} \mathrm{C}$ ), as well as blocks of meat produced in Brazil, Paraguay, Uruguay. The freezing of fresh and chilled meat was carried out in accordance with the instructions on refrigeration and storage of meat in the meat industry. Frozen meat was stored at a temperature of minus $20^{\circ} \mathrm{C} \pm 2^{\circ} \mathrm{C}$ [2]. Defrosting of meat samples was carried out in a steam-air environment at a temperature of plus $18^{\circ} \mathrm{C} \pm 2{ }^{\circ} \mathrm{C}$ and an average air velocity of $0.5-1.0 \mathrm{~m} / \mathrm{s}$ in an industrial manner under the conditions of "Klinsky meat-packing Plant".

\section{Reagents}

All reagents used in this study were of analytical grade of purity or higher. To perform the research, reference substances of high purity were used: free nucleotides-ATP, ADP, AMP, IMP and nucleosides-inosine and hypoxanthine (the substance content in preparations from 99.96\%, manufactured by "SIGMA", USA).

The free meat nucleotides were extracted according to a standard procedure for extracting adenine-containing nucleotides. The weighed muscle tissue was mixed with perchloric acid solution $(0.6 \mathrm{M})$ and thoroughly triturated, then the resulting mixture was centrifuged, the precipitate was removed and in the re- 
sulting supernatant the $\mathrm{pH}$ of the medium was neutralized with a solution of potassium hydroxide (0.1 M KOH) [12].

The free nucleotides, nucleosides and composition were studies by high-performance liquid chromatography (HPLC) and spectrophotometric (SF) method.

The content of free nucleotides and nucleosides in meat samples was determined on Agilent Technologies (USA) "Agilent-1200" liquid chromatograph on a Phenomenex Sphere Clone ODS (2) column $250 * 4 / 6.5 \mu \mathrm{m}$.

Analysis of the UV-spectra was carried out on mono-solutions, mixtures of solutions of pure free nucleotides in concentrations characteristic for fresh and cooled meat, and on extracts of free nucleotides obtained from meat. The measurements were carried out using spectrophotometer "SPEKOL-1500 "Analytik, JENA (Germany) and specialized software "Win ASPECT", as well as on the spectrophotometers from other manufacturers-Cary 50 Bio "Agilent Technologies" (USA), UV-3600 Shimadzu (Japan).

Comparative analysis of the adequacy of data obtained by SF method and the HPLC were performed.

\section{Statistical methods}

The experiments were comprised in six series of triplicate measurements each. The results were processed statistically with programs OriginPro v.8.1 for Windows (OriginLab, Data Analysis Graphing Software, USA) and Statistica for Windows, v.5.0 (StatSoft Inc., USA) and evaluation of statistical significance was done by the Student's $t$ test with $\mathrm{p} \leq 0.05$.

\section{Results and Discussion}

\subsection{Studies of the Composition and Content of Free Nucleotides by HPLC}

Free nucleotides are low-molecular nitrogen-containing compounds found in tissues both in the free state and in the composition of nucleic acids. Nucleotides differ from each other in their constituent nitrogenous bases and different degrees of phosphorylation. Of all the nucleotides, mono-, di- and triphosphates of adenosine monophosphate (AMP, ADP, ATP) and-inosine monophosphate (IMP), inosine and hypoxanthine, the recent increase in concentration during the ripening and storage of meat are present in the largest number of tissues in the tissue. During the ripening and storage of meat, the transformation of nucleotides goes to the stage of formation of free nitrogenous bases and their derivatives. The quantitative content, nature and rate of change in the qualitative composition of nucleotides in meat significantly influence the formation of physicochemical properties and qualitative characteristics of meat raw materials, its nutritional and biological value. Rigid meat is hard exposed to proteolytic ferments of the gastrointestinal tract, the digestibility of meat is reduced [1] [5] [13]. To determine nucleotides and nucleosides in experimental meat samples (frozen fresh and after cooling) using the HPLC method, solutions of reference 
free nucleotides and nucleosides identical in their content in meat were obtained and their optical properties were studied. The results of determining the qualitative composition and quantitative content of free nucleotides of reference substances of high purity are presented in chromatograms (Figure 1).

It was found that the ATP solution has a characteristic absorption maximum at a wavelength of $260 \mathrm{~nm}, \mathrm{ADP}$ at $259 \mathrm{~nm}, \mathrm{AMP}$ at $260 \mathrm{~nm}, \mathrm{IMP}$ at $250 \mathrm{~nm}$, inosine at $250 \mathrm{~nm}$, hypoxanthine at $249 \mathrm{~nm}$. It should be noted that substances containing adenosine-ATP, ADP, AMP have an absorption maximum in the wavelength range of 259 - $260 \mathrm{~nm}$, inosine and hypoxanthin-at 249 - $250 \mathrm{~nm}$.

The results of determining the qualitative composition and quantitative content of nucleotide of meat frozen in a fresh form or after cooling are presented in chromatograms (Figure 2) and Table 1.

From the chromatogram (Figure 2, on the left) it follows that the main content of free nucleotides of meat frozen in a fresh form is ATP. During cooling, ATP almost completely passes into the IMP, as can be seen from the chromatogram

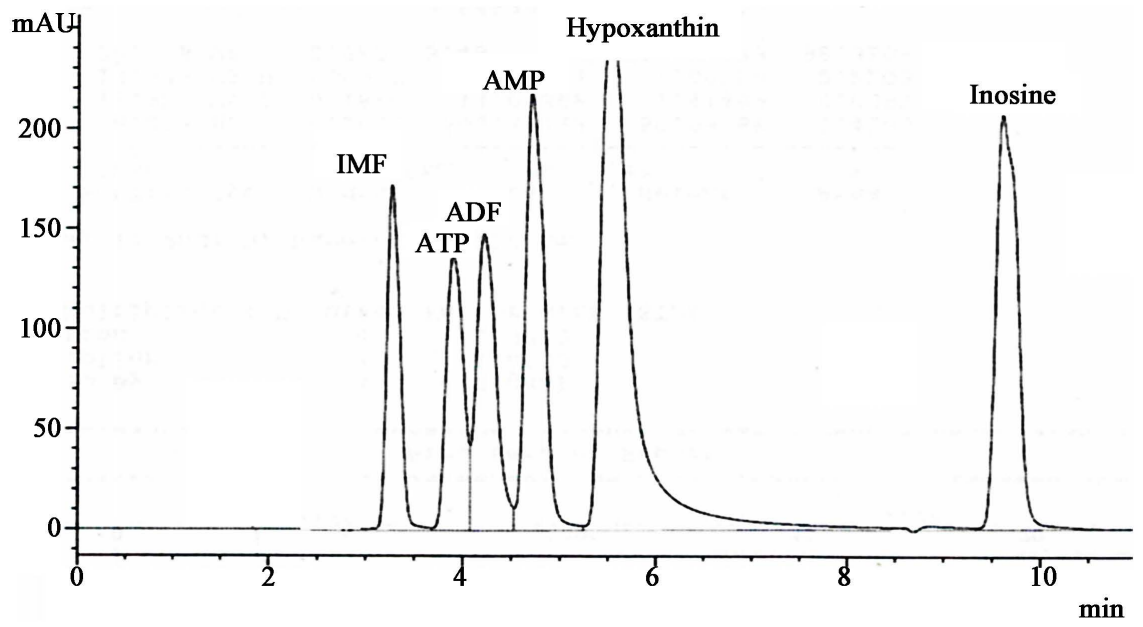

Figure 1. Chromatogram of separation of reference substances of high purity: ATP, ADP, AMP, IMP, inosine and hypoxanthine. IMP-inosine monophosphoric acid (inosinic acid); ATP_adenosine triphosphoric acid; ADP_adenosine diphosphoric acid; AMP_adenosine monophosphoric acid.

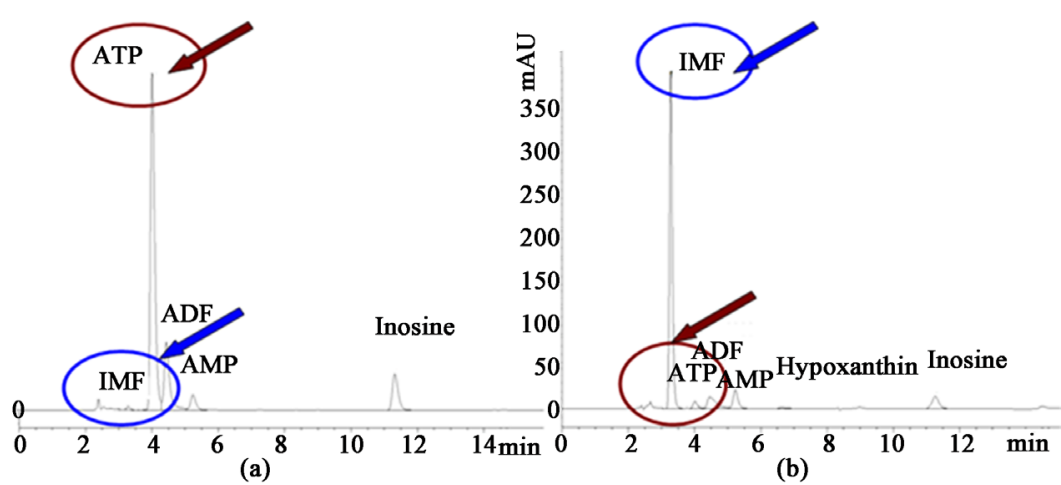

Figure 2. Chromatograms of separation of free nucleotides and nucleosides of muscle tissue, (a) meat frozen in a fresh form; (b) meat frozen after cooling. 
Table 1. Content of free nucleotides and nucleosides of meat frozen in a batch form and after cooling.

\begin{tabular}{ccc}
\hline \multirow{2}{*}{$\begin{array}{c}\text { Free nucleotides and } \\
\text { meat nucleosides }\end{array}$} & Content of free nucleotides and nucleosides in frozen meat, $\mu \mathrm{mol} / \mathrm{g}$ \\
\cline { 2 - 3 } ATP & in the fresh form $(\mathrm{x} \pm \mathrm{s})$ & after cooling $(\mathrm{x} \pm \mathrm{s})$ \\
ADP & $3.48 \pm 0.5$ & $0.16 \pm 0.14$ \\
AMF & $1.40 \pm 0.55$ & $0.25 \pm 0.21$ \\
IMF & $0.32 \pm 0.22$ & $0.18 \pm 0.15$ \\
Inosine & $0.44 \pm 0.29$ & $5.39 \pm 1.26$ \\
Hypoxanthin & $0.22 \pm 0.19$ & $0.15 \pm 0.12$ \\
\hline
\end{tabular}

for meat frozen after cooling (Figure 2, on the right). Therefore, for the identification of meat raw materials of various methods of freezing, it was suggested to use the quantitative ratio of nucleotides ATP to IMP.

From the data in Table 1, it follows that in meat frozen in a fresh form, the ATP content is 21.8 times higher, and the amount of IMF is 12.3 times lower than in meat frozen after cooling. As meat is stored, the content of free nucleotides decreases rather quickly, and the content of hypoxanthin gradually increases. The content of ATP falls particularly fast. This is due to the fact that ATP - a very unstable compound and immediately after the slaughter of the animal stops its resynthesis. The rate of decay of nucleotides depends on the storage temperature, and these changes are especially pronounced in the content of ATP and IMP in paired meat compared to the cooled one [5] [13].

Thus, it is confirmed that the meat frozen in a fresh form has characteristic differences in the quantitative content of nucleotides from meat frozen after cooling, which allows them to be differentiated by high-performance liquid chromatography using the ratio of ATP concentration to IMP. However, this method is not applicable for production control, requires a long time of analysis, highly qualified personnel and significant material costs.

\subsection{Spectrophotometric Studies of Solutions of Reference Substances of Free Nucleotides and Nucleosides}

The spectrophotometric method for determination of adenine nucleotides is based on the measurement of light absorption in the ultraviolet region. Solutions of nucleotides have characteristic absorption peaks at the wave lengths 260 and $250 \mathrm{~nm}$ determined by the content in the composition of the molecules ADP and inosine group respectively. After conducting a series of spectrophotometric studies, which has allowed to reveal characteristic signs, allowing to differentiate the analyzed solutions of substances are nucleotides and nucleosides, were carried out spectrophotometric studies directly to raw meat.

Spectrophotometric data on the studies of mono-solutions of chemically pure substances ATP, ADP, AMP, IMP, inosine and hypoxanthine (the dependence of the optical density $\chi$ on the wavelength $\lambda$ ) are shown in Figure 3. 


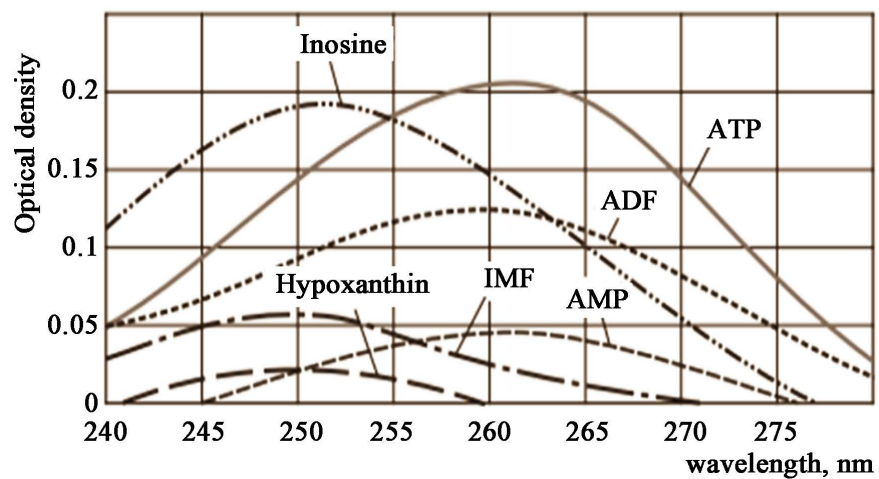

Figure 3. Characteristic absorption spectra of mono-solutions of free nucleotides and nucleosides.

The results of studies of mixtures of solutions of free nucleotides and nucleosides in concentrations identical for their content for meat frozen in a fresh form and after cooling are shown in Figure 4.

Data on the quantitative content of individual components for the preparation of solutions were obtained on the basis of the studies carried out using the HPLC method (Table 1).

It is determined that the maximum absorption spectrum of a mixture of free nucleotides and nucleosides identical in content to meat frozen in a paired form is observed in the wavelength range $259 \pm 0.6 \mathrm{~nm}$, characteristic of adenosine nucleotides-ATP, ADP, AMP, while, the height of the maximum absorption spectrum of a mixture of free nucleotides and nucleosides identical in content to meat frozen after cooling, falls within the wavelength range $249 \pm 0.5 \mathrm{~nm}$ (Figure 4), characteristic of inosic acid and nucleosides.

The maxima of the absorption spectra of mixtures of free nucleotides and nucleosides that are adequate for their content in meat frozen in a fresh or after cooling are significantly different from each other when $\mathrm{p}<0.01, \mathrm{n}=20$.

To ensure the objectivity of estimating free-nucleotide solutions identical in their content in the two above-mentioned frozen meat variants, taking into account the revealed specific differences and the existing relationship between the ratio of ATP optical density at $260 \mathrm{~nm}$ to the optical density of IMP at $250 \mathrm{~nm}$ and the transition of ATP to IMP, we proposed the M-index, which makes it possible to identify the test solutions. The proposed $\mathbf{M}$-index is calculated as the ratio of the optical density:

$$
\mathbf{M}=O D_{260} / O D_{250}
$$

where, $M$ is the ratio of the optical density measured at $260 \mathrm{~nm}$ to the optical density measured at $250 \mathrm{~nm}$. The determination of the $\mathrm{M}$-index in the solutions studied was carried out in six fold repetition for each group of solutions. The results obtained are presented in Table 2.

It has been established that the values of the ratio of the optical density ratio $\mathbf{M}=O D_{260} / O D_{250}$ for solutions identical in content of free nucleotides and nucleosides of meat frozen in a fresh form are more than unity and range from 


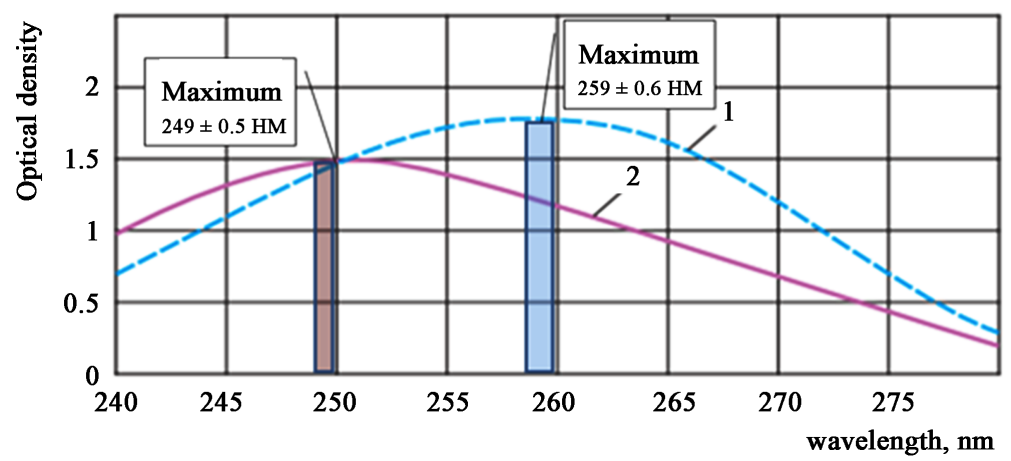

Figure 4. Spectra of mixtures of free nucleotides and nucleosides of high frequency, imitating fresh (1) and chilled (2) meat.

Table 2. Optical density of solutions of free nucleotides and nucleosides identical in their content in meat frozen in a fresh form and after cooling and index $\mathrm{M}$.

\begin{tabular}{ccccccc}
\hline & \multicolumn{2}{c}{$\begin{array}{c}\text { Solutions of free nucleotides and } \\
\text { nucleosides identical in content } \\
\mathbf{N}^{*}\end{array}$} & $\begin{array}{r}\text { in meat frozen in a fresh form } \\
\mathrm{OD}_{260}\end{array}$ & $\mathrm{OD}_{250}$ & $\mathrm{M}$-index & \multicolumn{2}{c}{$\begin{array}{c}\text { Solutions of free nucleotides } \\
\text { and nucleosides identical in } \\
\text { content in meat frozen after cooling }\end{array}$} \\
\cline { 2 - 7 } & 1.47 & 1.27 & 1.16 & 0.87 & 1.2 & 0.73 \\
\hline 1 & 1.43 & 1.3 & 1.1 & 0.85 & 1.17 & 0.73 \\
2 & 1.57 & 1.43 & 1.1 & 0.85 & 1.19 & 0.71 \\
3 & 1.56 & 1.41 & 1.11 & 0.9 & 1.21 & 0.74 \\
5 & 1.52 & 1.39 & 1.09 & 0.89 & 1.19 & 0.75 \\
6 & 1.53 & 1.38 & 1.11 & 0.97 & 1.35 & 0.72 \\
$\pm \mathrm{s}$ & $1.51 \pm 0.05$ & $1.36 \pm 0.06$ & $1.11 \pm 0.02^{*}$ & $0.89 \pm 0.04$ & $1.22 \pm 0.07$ & $0.73 \pm 0.01^{*}$ \\
\hline
\end{tabular}

Differences are reliable by the Student's test for the indicator $\mathrm{M}$ of the compared solutions at $\mathrm{p}<0.01$.

1.09 to 1.16 , and for solutions identical in terms of their the content of meat frozen after cooling, index $\mathrm{M}$ is less than one and is in the range from 0.71 to 0.75 .

Thus, the established values of the $\mathrm{M}$ indicator for the solutions under study are significantly different at a significance level of $p<0.01$, which allows one to justify the choice of this parameter for characterizing the thermal state of meat frozen in a fresh form or after cooling.

The results of studies of mixtures of solutions of free nucleotides and nucleosides in concentrations content for meat frozen in a fresh form and after cooling are shown in Figure 5.

It is determined that the maximum absorption spectrum of a mixture of free nucleotides and nucleosides content to meat frozen in a paired form is observed in the wavelength range $259 \pm 0.8 \mathrm{~nm}$, while, the height of the maximum absorption spectrum of a mixture of free nucleotides and nucleosides content to meat frozen after cooling, falls within the wavelength range $249 \pm 0.6 \mathrm{~nm}$ (Figure 5). 


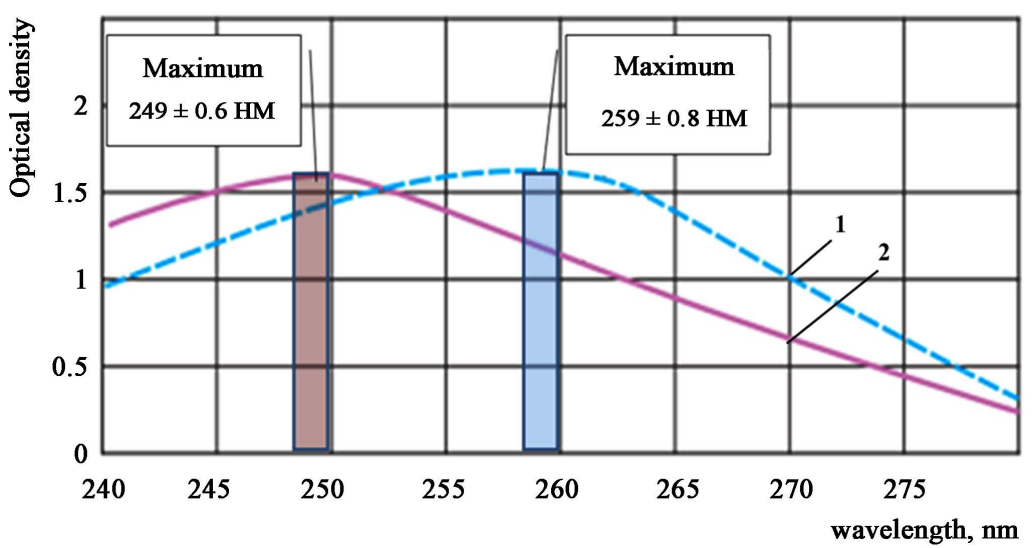

Figure 5. Spectra of extracts of free nucleotides and nucleosides of meat frozen in a fresh form (1) and after cooling (2).

The value of $\mathrm{M}$ for extracts of free nucleotides obtained from meat frozen in a fresh form $(1.10 \pm 0.01)$ is 1.5 times higher than the value of $\mathrm{M}$ for meat frozen after cooling $(0.73 \pm 0.02)$. The difference between the determinants is reliable at a significance level of $p<0.01$, which makes it possible to identify the meat being examined.

The results of studies on the identification of the thermal state of frozen boneless meat cuts of different countries of production, determined by the value of the $\mathrm{M}$ indicator, are given in Table 3.

A comparative analysis of frozen boneless cuts from Brazil, Paraguay and Uruguay in terms of the value of $\mathbf{M}$-index showed that for all samples the average values of $\mathrm{M}$ are in the range from 0.73 to 0.78 with the maximum deviation from 0.71 to 0.78 , which is a proof their freezing after cooling. The advantages of using a single-phase freezing method are also confirmed by the work of foreign experts in the study of the structure, chemical structure of muscles and their effect on the technological characteristics of meat [14].

\subsection{Assessment of the Reliability of Data on the Identification of Meat Frozen in a Batch or after Cooling, Obtained by HPLC and SF Method}

Results of studies of meat, frozen immediately after slaughter (meat fresh, fresh form) and after cooling, using a HPLC method and the method developed SF (Table 4) show the adequacy of the experimental data obtained by both methods.

\section{Conclusions}

The experimental results obtained make it possible to draw the following conclusions:

- a qualitative composition and quantitative content of free nucleotides of meat frozen in a fresh form and after cooling by HPLC was experimentally established. 
Table 3. Values of $M$ index in extracts of free nucleotides and nucleosides of frozen boneless meat cuts (LMOs) from different countries of production.

\begin{tabular}{cccc}
\hline $\mathbf{N}^{\bullet}$ & Brazil & Paraguay & Uruguay \\
\cline { 2 - 4 } & M-index & M-index & M-index \\
\hline 1 & 0.78 & 0.71 & 0.78 \\
2 & 0.72 & 0.78 & 0.77 \\
3 & 0.71 & 0.76 & 0.75 \\
4 & 0.73 & 0.77 & 0.73 \\
5 & 0.71 & 0.75 & 0.75 \\
6 & 0.73 & 0.76 & 0.78 \\
$\mathrm{x} \pm \mathrm{s}$ & $0.73 \pm 0.03$ & $0.76 \pm 0.02$ & $0.76 \pm 0.02$ \\
\hline
\end{tabular}

Table 4. Comparative analysis of the adequacy of data obtained by HPLC and the SF method

\begin{tabular}{|c|c|c|c|c|}
\hline \multirow{4}{*}{$\mathbf{N}^{\bullet}$} & \multicolumn{2}{|c|}{ The HPLC method } & \multicolumn{2}{|c|}{ The SF method } \\
\hline & \multicolumn{2}{|c|}{ Concentration ratio ATP/IMP, $(\mu \mathrm{mol} / \mathrm{g})$} & \multicolumn{2}{|c|}{$\begin{array}{l}\text { Optical Density Ratio ATP/IMP, } \\
\qquad\left(\mathrm{OD}_{260} / \mathrm{OD}_{250}\right)\end{array}$} \\
\hline & \multicolumn{4}{|c|}{ Frozen meat } \\
\hline & in a fresh form & after cooling & in a fresh form & after cooling \\
\hline 1 & 13.92 & 0.06 & 1.11 & 0.72 \\
\hline 2 & 10.64 & 0.02 & 1.10 & 0.72 \\
\hline 3 & 11.92 & 0.04 & 1.10 & 0.71 \\
\hline 4 & 15.58 & 0.03 & 1.10 & 0.75 \\
\hline 5 & 22.11 & 0.06 & 1.08 & 0.73 \\
\hline $\mathrm{x} \pm \mathrm{s}$ & $14.84 \pm 4.48^{*}$ & $0.04 \pm 0.02^{*}$ & $1.10 \pm 0.01^{* *}$ & $0.73 \pm 0.02^{* *}$ \\
\hline
\end{tabular}

${ }^{*}$ Differences are reliable by the Student's test between the concentration ratios for the compared samples at $\mathrm{p}<0.01$; $^{* *}$ are reliable by the Student's test between the indicator $\mathrm{M}$ for the compared samples $\mathrm{p}<0.01$.

- to ensure the objectivity of the existing relationship between the ratio of the optical density of ATP to the optical density of the IMT along the wavelength of the maxima of the absorption spectra and the transition of ATP to the UTI, we proposed an index M that allows us to identify the thermal state of the meat.

- a spectrophotometric method for identifying meat has been developed to justify the choice of technological modes of its defrosting. The method was tested at the enterprise of JSC "Meat-packing plant Klinsky". The method was tested in the accredited laboratories of the VNIIMP them. VM Gorbatov and the VNITEK FGBNU.

The technical and economic assessment of the SF method and the HPLC method shows that: the cost of the equipment necessary to introduce the SF method is 11 times lower, and the analysis time is 6 times less. The C-method is simpler and does not require the training of highly qualified personnel, and the mea- 
surement results have comparable accuracy in assessing the thermal state of the meat.

The developed method for identifying meat frozen fresh and after cooling allows us to introduce an effective differentiated technology for thawing freshly frozen meat that prevents the appearance of the THAW RIGOR phenomenon in the case of defrosted boneless meat, which contributes to preserving the technological properties of meat raw material, its nutritional and biological value by reducing the mass loss during thawing in the form of flowing juice with the biologically active components dissolved in it, including proteins, amino acids, minerals, preserving the structural and mechanical properties of meat improves its digestibility by the gastrointestinal tract of man.

\section{References}

[1] Dibirisulaev, M.A., Belozerov, G.A., Limonov, G.E. and Khvilya, S.I. (2002) Scientific and Practical Aspects of the Prediction of "Rigidity-Thawing" and the Development of a New Technology of Meat Thawing. Storage and Processing of Agricultural Raw Materials, 2, 36-39.

[2] Belozerov, G.A. (2002) Practical Aspects of Technology Refrigeration Conservation. Refrigerating Business (Rus), 22-26.

[3] Jablonecko, L.A. and Zhiltsov, V.V. (2007) The Effect of Different Temperature Regimes on the Duration of the Freezing Process and Quality of Raw Meat. Fundamental Research, 2, 32-33.

[4] Farouk, M.M. and Swan, J.E. (1998) Effect of Rigor Temperature and Frozen Storage on Functional Properties of Hot-Boned Manufacturing Beef. Meat Science, 49, 233-247. https://doi.org/10.1016/S0309-1740(97)00134-4

[5] Aliani, M., Farmer, L.J., Kennedy, J.T., Moss, B.W. and Gordon, A. (2013) Post-Slaughter Changes in ATP Metabolites, Reducing and Phosphorylated Sugars in Chicken Meat. Meat Science, 94, 55-62. https://doi.org/10.1016/j.meatsci.2012.11.032

[6] Zbigniew, D. (2016) Book Review: Meat Quality-Genetic and Environmental Factors. Polish Journal of Food and Nutrition Sciences, 66, 147-150. https://doi.org/10.1515/pjfns-2016-0005

[7] Leygonie, C., Britz, T.J. and Hoffman, L.C. (2012) Impact of Freezing and Thawing on the Quality of Meat. Meat Science, 91, 93-98. https://doi.org/10.1016/j.meatsci.2012.01.013

[8] Adzitey, F. and Nurul, H. (2011) Pale Soft Exudative (PSE) and Dark Firm Dry (DFD) Meats: Causes and Measures to Reduce These Incidences-A Mini Review. International Food Research Journal, 18, No. 1.

[9] Silva, K.R.C. and Menão, M.C. (2015) Microbiological Assessment of Chicken Meat Cuts Sold in the City of São Paulo. Environmental Health Atlas, 3, 17-23.

[10] Dibirasulaev, M.A., Belozerov, G.A., Arkhipov, L.O., Shibanova, E.D. and Belyanchikova, I.V. (2016) Study of the Composition and Content of Free Nucleotides of Cattle Meet at Different Stages of Refrigeration Processing and Storage. Kholodilnaya Tekhnika, No. 2, 58-64.

[11] Ballin Ballin, N.Z. and Lametsch, R. (2008) Analytical Methods for Authentication of Fresh vs. Thawed Meat-A Review. Meat Science, 80, 151-158. https://doi.org/10.1016/j.meatsci.2007.12.024 
[12] Severin, S.E. (1989) Practical Work on Biochemistry: Textbook. Allowance. Severin, S.E. and Solovyov, G.A., Eds., MGU, Moscow, 509 p.

[13] Tikk, M., Tikk, K., Tørngren, M.A., Meinert, L., Aaslyng, M.D., Karlsson, A.H. and Andersen, H.J. (2006) Development of Inosine Monophosphate and Its Degradation Products during Aging of Pork of Different Qualities in Relation to Basic Taste and Retronasal Flavor Perception of the Meat. Journal of Agriculturwal and Food Chemistry, 54, 7769-7777. https://doi.org/10.1021/jf060145a

[14] Girolami, A., Napolitano, F., Faraone, D. and Braghieri, A. (2013) Measurement of Meat Color Using a Computer Vision System. Meat Science, 93, 111-118.

https://doi.org/10.1016/j.meatsci.2012.08.010 